\title{
REPRODUCTIVE VERSATILITY IN RUBUS
}

\section{RASPBERRY-BLACKBERRY HYBRIDS}

M. B. CRANE

John Innes Horticultural Institution, Bayfordbury, Hertford, Herts

and P. T. THOMAS

Department of Agricultural Botany, University of Wales, Aberystwyth

With an appendix by

C. D. DARLINGTON

Received I.v.48

THE Veitchberry $4^{x}=28$ and the Mahdiberry, $3 x=2 \mathrm{I}$, were both raised from a cross between the hedgerow Blackberry Rubus rusticanus, $2 x=14$, and tetraploid forms of the raspberry Rubus idaeus, $4 x=28$. No diploid hybrid, the result of crossing diploid forms of these two species, appears to be known and attempts we have made to cross them have entirely failed. It therefore seems that seeds are formed only when an unreduced germ cell from one or both diploid parents takes part in fertilisation or when a polyploid form of $R$. idaeus is one of the parents. That unreduced germ cells occasionally occur in $R$. rusticanus is evident, not only from the Veitchberry, but also from the origin of the John Innes Berry, $4 x=28$, which was derived from $R$. rusticanus, $2 x=14$, crossed $R$. thyrisiger, $4 x=28$ (Crane and Darlington, 1927).

In 1935 a plant of the Mahdiberry growing at Merton sent up a very vigorous basal shoot which was found to be pentaploid $5 x=35$. This shoot was removed, but although care was taken it was not possible to be certain that it was attached to the original Mahdi. It is thus derived either as somatic mutation or by segregation in a seedling. Plants derived vegetatively from this shoot are much more vigorous, produce larger fruits and when provision is made for crosspollination they fruit more freely (fig. I). The undersides of the leaves of the pentaploid are not so densely hairy as those of the triploid, but apart from this and the increase in size, which is proportionate in all organs, they seem indistinguishable.

Most cultivated varieties of raspberry are heterozygous. Thus the variety Lloyd George with red fruits, red prickles, and hairy growth, gives seedlings with yellow fruits, green prickles, and glabrous growth when selfed. Similarly, the red-fruited variety Superlative with hermaphrodite flowers, in addition to giving yellow fruits, also gives male and female forms by segregation of recessive genes (Crane and Lawrence, I93I). Other genes which suppress pollen development also occur in raspberries (Lewis, I939). 
The Veitchberry combines the characters of its parent species. For example its fully developed leaves are partly pinnate as in $R$. idaeus and partly palmate as in $R$. rusticanus. Upon selfing, its offspring vary only in minor respects such as the colour of the petals and prickles; there is no approach to either of the parental forms. Upon crossing with $R$. idaeus and other species, the behaviour of the Veitchberry is again typically that of a species, but exceptionally a few aberrant forms have appeared in the progeny. All this suggests the general suppression of segregation, as in Primula kewensis, through the pairing of like chromosomes.

There are two forms of the Veitchberry; they differ in pollen development, one produces pollen abundantly, the other very sparsely and gives a proportion of the first form on selfing. Whether this difference arose somatically or sexually is not clear, but it is no doubt due to the action of the pollen-inhibiting genes found in the raspberry parent.

From crossing the Veitchberry as female with two diploid forms of R. idaeus: (I) the variety Lloyd George and (2) a male seedling from the variety Superlative, we raised two families, one of I 8 , the other of 126 seedlings. In both families variation occurred in petal and prickle colour and in other minor respects. But, apart from this variation, the result of the heterozygosity of the raspberry parent, 237 out of the 244 seedlings ( 97.2 per cent.) were intermediate between the parents, and of a uniform type in habit of growth and other major characters.

They were all highly sterile.

Several of them were cytologically examined and found to be triploid, $3^{x}=2 \mathrm{I}$.

The others, four plants in one family and three in the other were strikingly different. They were all of reduced size. Three of these are shown in plate . BB was very similar to its original blackberry grandparent $R$. rusticanus. RR was typically $R$. idaeus. $\mathrm{RB}$ was intermediate between these two species; it was like the Veitchberry but with slenderer and less robust growth, see fig. 2. The other four plants were three blackberry and one intermediate. The raspberry-like seedling was fertile, the others infertile, some of the blackberries entirely so.

The three illustrated seedlings were found to be diploid $2 x=\mathrm{I} 4$ and the other four must have been the same.

To elucidate the origin of these exceptional plants and assess the chromosome relationship between blackberry and raspberry, one of us (P.T.T.) examined the plant RB, the Veitchberry, and other allied Rubi shown below.

$$
\begin{aligned}
& \text { Veitchberry } \times \text { Lloyd George } 2 x=14 \text {. . RB } \\
& \text { Veitchberry } \times \text { Lloyd George } 3 x=2 \mathrm{I} \text {. . RRB } \\
& \text { Auto-triploid Raspberry } 3^{x}=21 \text {. . . . RRR } \\
& \text { Mahdiberry } 3^{x}=2 \mathrm{I} \text {. . . . RRB }
\end{aligned}
$$




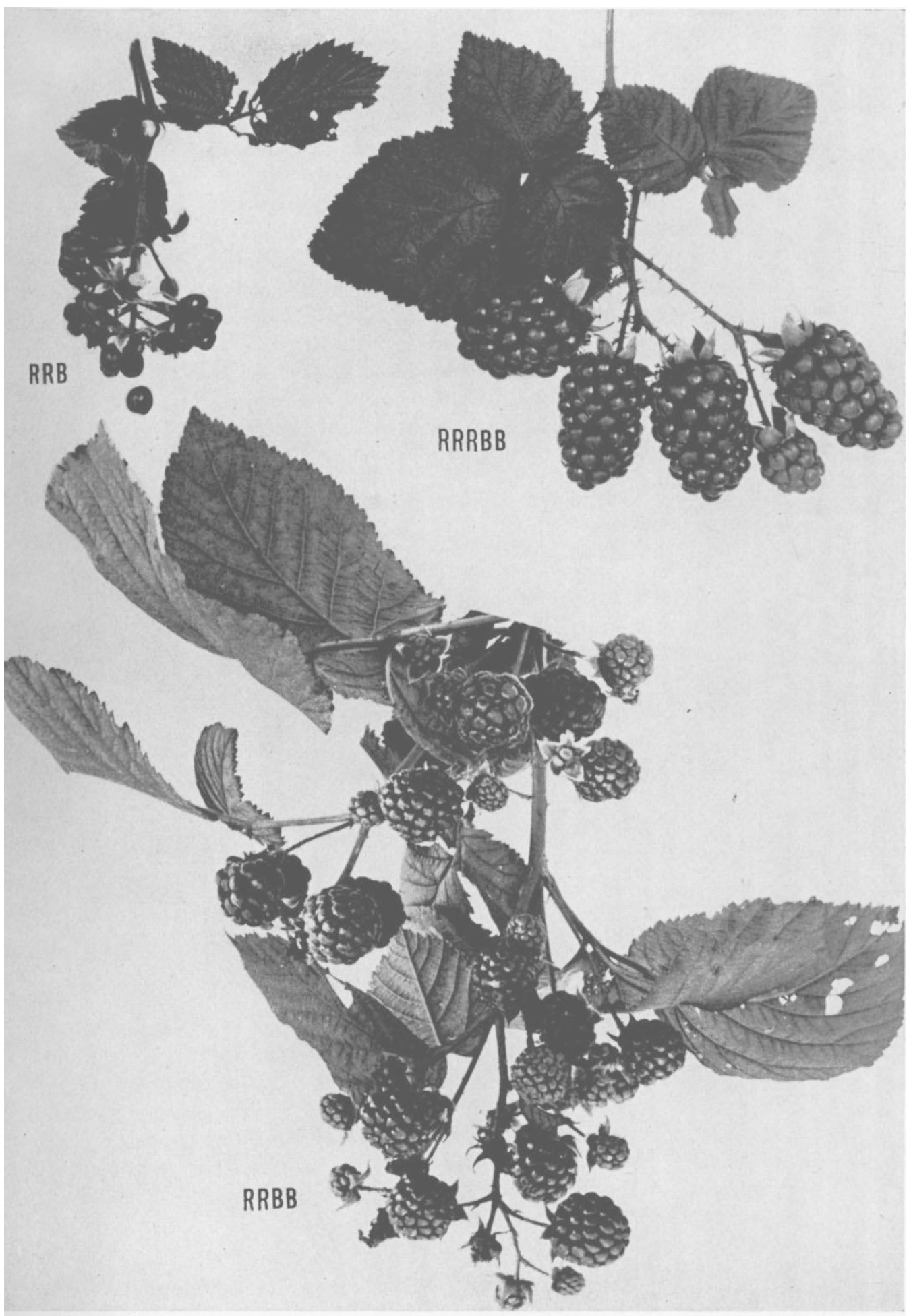

FIG. 1.-The triploid Mahdi RRB, pentaploid Mahdi RRRBB and the tetraploid Veitchberry RRBB. Reduced to $\frac{2}{3} \cdot$ ? 
\begin{tabular}{ll} 
Auto-tetraploid Hailshamberry $4 x=28$ & RRRR \\
Veitchberry $4 x=28$, pollen sparse & RRBB \\
Veitchberry selfed $4 x=28$, pollen abundant & R \\
\hline
\end{tabular}

The Veitchberry used in these studies was the sparse pollen form. The anthers usually fail to develop and those that do develop more or less normally contain only a small proportion of germ tissueisolated islands of pollen mother-cells are found amongst the somatic tissue. Evidently some physiological disturbance in the young differentiating anther causes some of the potential mother-cells to remain as somatic cells. The degree and type of chromosome pairing did not materially differ from that of the form with normal anther and pollen development which was also examined.

Chromosome association at meiosis in these two plants and in the auto-tetraploid raspberry Hailshamberry, are compared in table $\mathrm{I}$.

TABLE I

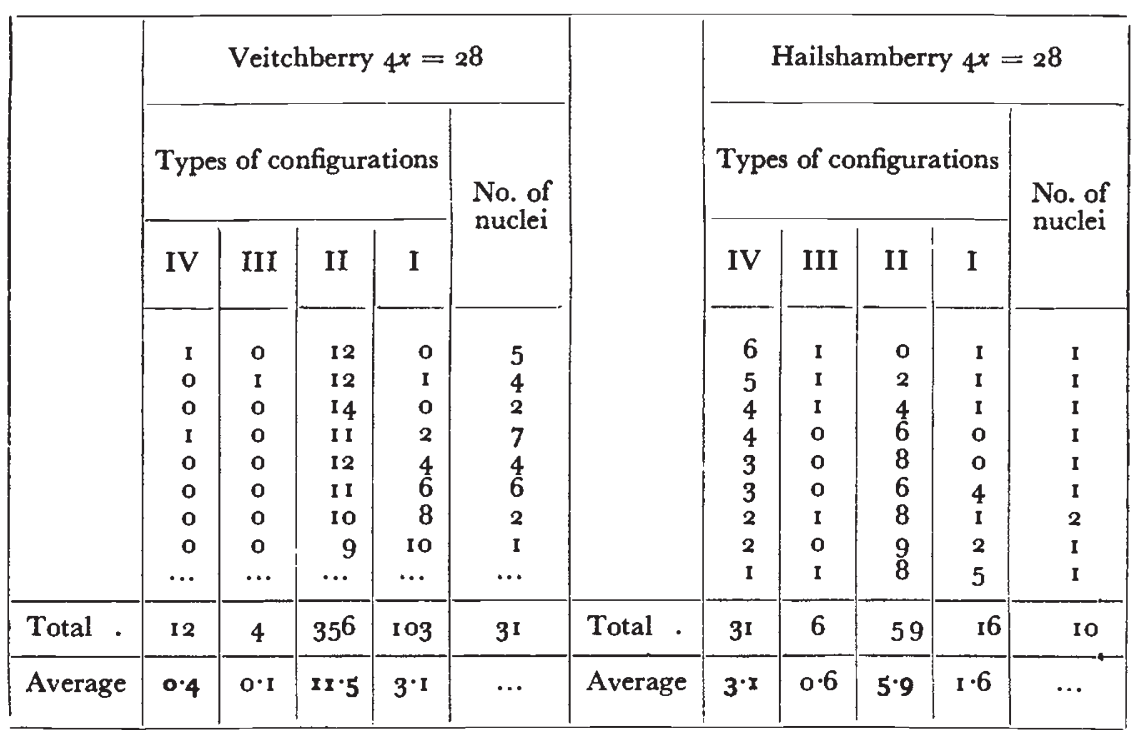

It seems that the Veitchberry differs cytologically from the autotetraploid raspberry in three respects: (I) the number of quadrivalents is lower, 0.4 per nucleus in the Veitchberry, compared with 3.1 per nucleus in the raspberry, (2) the number of univalents is higher and (3) the range in number of univalents is also much higher in the Veitchberry than in the tetraploid raspberry.

Little is known of the way in which raspberry and blackberry chromosomes have become differentiated from one another, because no direct diploid hybrid between the two species has been produced and available for study. The information we have, has been obtained somewhat indirectly from the study of polyploid hybrids. For example in the loganberry, $R$. loganobaccus, which we have shown to have 
two sets of raspberry and four sets of blackberry chromosomes, there is little if any pairing between the raspberry and blackberry chromosomes (Crane and Thomas, 1940). On the other hand in the Madhiberry the degree of chromosome association is only slightly less than in an autotriploid raspberry (table 2).

TABLE 2

Frequencies of cells with different numbers of bivalents or trivalents at meiosis in diploid and triploid forms of Rubus

\begin{tabular}{|c|c|c|c|c|c|c|c|c|c|c|c|}
\hline Plant & Formula & 0 & 1 & 2 & 3 & 4 & 5 & 6 & 7 & Total & Average \\
\hline $\begin{array}{l}\text { Veitchberry } 2 x \\
\text { Mahdiberry } 3^{x} \\
\text { Raspberry } 3^{x}\end{array}$ & $\begin{array}{l}\text { RB (II) } \\
\text { RRB (III) } \\
\text { RRR (III) }\end{array}$ & $\begin{array}{c}\cdots \\
7 \\
\cdots\end{array}$ & \begin{tabular}{|c|}
3 \\
5 \\
$\cdots$
\end{tabular} & $\begin{array}{r}2 \\
11 \\
2\end{array}$ & $\begin{array}{r}7 \\
\times 5 \\
5\end{array}$ & $\begin{array}{l}\text { I3 } \\
\text { IO } \\
\text { II }\end{array}$ & $\begin{array}{r}16 \\
6 \\
x 9\end{array}$ & $\begin{array}{r}10 \\
6 \\
13\end{array}$ & $\begin{array}{r}3 \\
6 \\
10\end{array}$ & $\begin{array}{l}54 \\
66 \\
60\end{array}$ & $\begin{array}{l}4 \cdot 5 \\
3 \cdot 3 \\
5 \cdot 0\end{array}$ \\
\hline
\end{tabular}

It, therefore, appears that raspberry and blackberry chromosomes are sufficiently differentiated to compel self-pairing when there are two sets of each in the hybrid but, when there is no opportunity for differential pairing as in the diploid, and to a lesser extent in triploids, the two types of chromosomes can pair with very little restriction.

Chromosome pairing in the Veitchberry is not that expected of a raspberry-blackberry tetraploid hybrid. Quadrivalents should be less frequent than in the auto-tetraploid but the wide range in number of univalents is not characteristic of an allo-tetraploid. We, therefore, suggest that the failure of pairing is genotypically controlled.

The exceptional diploid plants RB which occurred in the family Veitchberry $\times$ Lloyd George Raspberry enabled us to test this conclusion (table 2). There chromosome association ranges from complete pairing to almost complete failure of pairing. This kind of behaviour is what has been observed in pure species where the failure of pairing is due to the presence of a single gene. This interpretation would agree with our earlier conclusion that chromosome pairing in the Veitchberry itself is subject to genic interference.

The seven anomalous diploids must all have originated from the embryo sac of the Veitchberry, the male taking no part in fertilisation. In the BB plants it seems that the chromosomes from the Veitchberry were exclusively blackberry, in $R R$ exclusively raspberry and in RB presumably one set of each.

Regular bivalent formation with autosyndesis (self-pairing) would give embryo sacs with the chromosome constitution RB. Haploid parthogenesis with this type of chromosome behaviour would thus lead to the intermediate type of plant. Segregation to give rise to embryo sacs of the constitution RR or BB for all seven chromosomes would demand complete quadrivalent formation with an orientation at the first metaphase of meiosis, such as would occur only once in I 28 times. But our analysis of chromosome behaviour shows that 

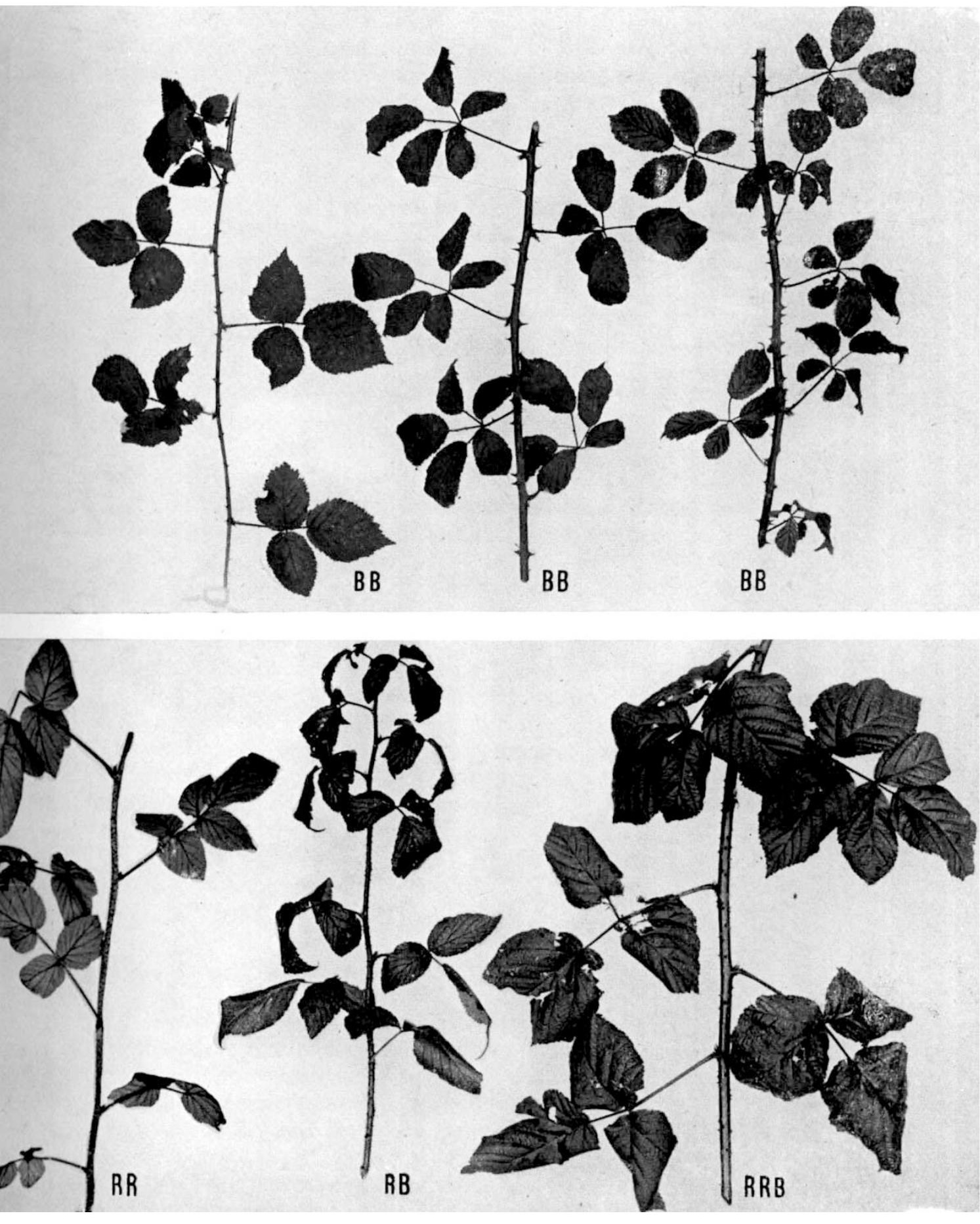

Frg. 2.-Diploid blackberries $B B$, diploid raspberry $R R$, diploid intermediate $R B$ and sexual triploid RRB, derived from Veitchberry RRBB crossed raspberry RR. Reduced to $\frac{3}{26}$. 
quadrivalent formation is infrequent in the Veitchberry. Alternatively we may visualise the origin of the $B B$ and $R R$ embryo sacs as being derived from a random separation of unpaired chromosomes where there has been a high proportion of univalents.

\section{SUMMARY}

I. The Veitchberry $4 x=28$ was derived from $R$. rusticanus $2 x=\mathrm{I} 4$ and a tetraploid form of $R$. idaeus $4 x=28$. Upon selfing there is no approach to either parental forms, it breeds true to its intermediate character.

2. Upon crossing with $R$. idaeus the behaviour of the Veitchberry is again typically that of a species, but exceptional diploid forms have appeared in the progeny : four blackberry-like, two intermediate but slender and one raspberry-like.

3. The chromosomes of the blackberry and the raspberry are differentiated and normally pair among themselves when two sets of each are present in the hybrid.

4. With less opportunity for differential pairing as in the Mahdiberry $3 x=2 \mathrm{I}$ the chromosomes of the two types can pair effectively with one another.

5. The allotetraploid Veitchberry behaves exceptionally in that there is considerable failure of pairing. This failure appears to be due to genotypic control since no structural differences were observed between the raspberry and blackberry chromosomes.

6 . One of the intermediate diploid plants from the Veitchberry similarly behaved like an asynaptic diploid.

7. It is concluded that seven diploids in the progeny from Veitchberry $4 x=28$ crossed raspberry $2 x=14$ have originated parthenogenetically from the embryo sacs of the Veitchberry.

\section{APPENDIX}

\section{ON AN INTEGRATED SPECIES DIFFERENCE}

\section{D. DARLINGTON}

The families examined by Crane and Thomas from the cross of Veitchberry by raspberry fall into two classes, the sexual and the asexual. If these classes are separated and compared two apparently contradictory conclusions follow.

In the first place there is a segregation of species differences in the diploid asexual progeny which is evidently genuine. But the three types of diploid are those expected only if the raspberry-blackberry difference is behaving as a unit in inheritance. Now this difference, as the diagram shows, is an elaborate one. It must obviously depend on numerous mutually adapted gene changes. The recombination of 
these in diploids is prevented in the European flora, both wild and cultivated, by the elimination of all diploid hybrids. We now see that it is also prevented in polyploids by some other, even more fundamental, condition. This condition must be the integration of all the differences as a block within which no crossing-over occurs.

Diagram to show the contrasted effects of segregation on the raspberry-blackberry difference in sexual and asexual families of the hybrid

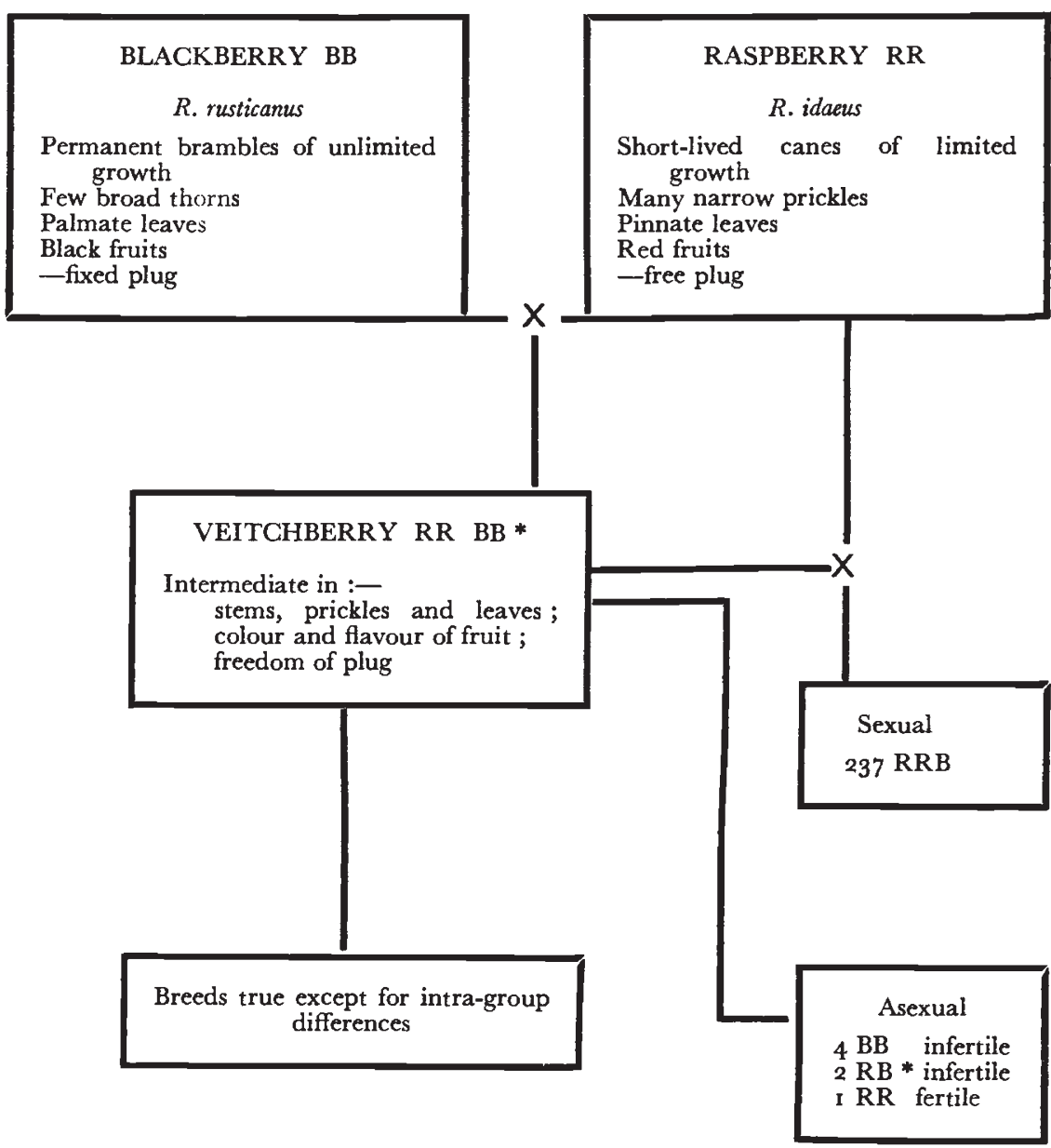

* Genotypic failure of pairing at meiosis in pollen mother cells

In the second place the eggs of the Veitchberry in sexual families (selfed as well as crossed) show no segregation of the differences between its parental species. If such segregation occurs the homozygotes are eliminated. The eggs in asexual families on the contrary show, not merely segregation, but an excess of homozygotes : it is the heterozygotes that are eliminated. 
This apparent contradiction is resolved by the cytological evidence. The frequency with which any one chromosome of the seven types enters into a quadrivalent in the Veitchberry is $0.39 / 7$ or 5.5 per cent. of the cells. A quadrivalent will give $R R-B B$ segregation in about half these cells and of them half will suffer loss of laggards and die, leaving 0.7 per cent. of RR gametes and 0.7 per cent. of $B B$, or one in 140 .

Thus one in $140^{2}$, or about 20,000 , selfed seedlings of Veitchberry should show segregation of each of the pure types, a possibility which has not been tested.

On the other hand the 0.7 per cent. segregation will be enhanced in the asexual families by the elimination of the diploid heterozygotes. This elimination is independantly shown by the failure of crosses between the diploid species-except in the production of polyploids such as the Veitchberry and the Mahdi. Thus the diploid elimination conceals the heterozygotes and the tetraploid recombination conceals the homozygotes.

The physiological integration of the raspberry and blackberry types in the Rubus cell must be combined with the basis of genetic isolation between the two groups. This isolation appears to be twofold: it is shown by the non-viability of direct diploid hybrids and also by the sterility of the indirect diploid arising from parthenogenesis of the tetraploid. This sterility strongly expressed appears as morphological male-sterility, and weakly expressed as reduced pairing at meiosis ; in all gradations it is genotypically controlled.

The non-viability of the diploid hybrid is also clarified by the present experiment. It seems not to be inherent since the diploid $\mathrm{RB}$ heterozygote can be raised in this indirect way. It probably depends on an error in the embryo-endosperm-ovary relationship in the diploid first cross which is no longer completely effective as a means of eliminating the diploid progeny of the tetraploid hybrid.

One consequence of this unitary difference is in the interpretation of the Mahdi sport. The pentaploid has evidently arisen by doubling (vegetative or sexual) in the triploid followed by the loss of one chromosome set, i.e. seven complementary chromosomes taken at random from the 42 .

The maintenance of the same external form with such a change of chromosome complement was preposterous in terms of a dispersed difference : it becomes intelligible in terms of an integrated one. To suppose that the 2 to I raspberry-blackberry proportion of the original Mahdi might persist in its giant mutant as a 3 to 2 proportion, avoiding any external qualitative variation by a precise regulation for all seven chromosomes of the set was too much. But to suppose that such a change could take place for a single member of the set although astonishing, is not utterly unreasonable. It now therefore becomes necessary to suppose that extensive changes hitherto known only in the "vegetative regulation" of the mosses (Wettstein, 1924) 
may take place in mitotic chromosome numbers, subject to mechanical loss of chromosomes and physiological selection of cells.

How is the raspberry-blackberry difference constituted?

In Oenothera we know how interchange makes it possible to hold together in the interchange hybrids large or complex differences lying in the seven different chromosomes. But here there is no evidence of interchange. All the differences must therefore lie in one chromosome.

We have to visualise a single super-gene consisting of many parts with mutually adjusted effects. These parts must be recombinable within each group as has been shown both for the prickles of the blackberry by Crane and Darlington (1927) and of the raspberry by Lewis (1940). It is in this way that an enormous range of species has been produced in each group, limited in number indeed only by the supply of materials and names that will enable us to preserve and describe them. On the other hand in crosses between the two groups the ultimate differences are held together. The diploid blackberry segregates arising from the Veitchberry have not merely the general blackberry character ; they have the very prickles of their rusticanus grandparent.

Such a system can arise by the inversion of a chromosome segment. In most natural populations there are inversions floating which occasionally will chance to include (or, shall we say, collide with) groups of mutually adapted genes capable of becoming a focus of fruitful discontinuity. And the coincidence having occurred, the resulting super-gene will evolve in the way that has been described from its successive stages by Darlington and Mather (I949).

Thus, in a variety of ways, we have evidence of the basis of the chief genetic divarication within the genus Rubus. The fact that such an elaborate divarication is effective only at the diploid level shows that it is at this level that the long-term evolutionary processes have been at work, the polyploids being a very recent, indeed perhaps a post-glacial, novelty.

In the present super-gene difference we can already see two stages of development. The integration of a block by suppression of crossing-over, and the non-viability of the diploid hybrid, both prevent recombination. But the integration of the block must have come first since the non-viability of the hybrid between two groups automatically brings to an end this organised divergence.

The closest analogy to the present situation is in the speltoid and fatuoid complexes of the cereals. The difference is that these complexes have arisen in cultivated plants and in hexaploids. Moreover they distinguish smaller systematic groups and lead to no inviability in the hybrid. The Rubus super-gene is therefore presumably a much older complex.

The problem that now arises is thus to compare the character and scope of the discontinuity between different sections of the main raspberry and blackberry groups in different parts of the world. 


\section{REFERENCES}

CRANE, M. B. I940.

Reproductive versatility in Rubus I. Morphology and inheritance.

7. Genet. 40, Iog-1 18.

CRANE, M. B. 1940.

The origin of new forms in Rubus II. The loganberry, R. Loganobaccus Bailey.

7. Genet. 40, 129-1 40.

CRANE, M. B., AND DARLINGTON, C. D. 1927 .

The origin of new forms of Rubus.

Genetica 9, 241-278.

CRANE, M. B., AND LAWRENCE, W. J. C. I 93 I.

Inheritance of sex, colour and hairiness in the raspberry Rubus idaeus.

7. Genet. 24, 243-255.

DARLINGTON, C. D., AND MATHER, K. 1949 .

Elements of genetics. London : Allen and Unwin.

LEWIS, D. 1939 .

Genetical studies in cultivated raspberries. I. Inheritance and linkage.

7. Genet. 38, 367-379.

THOMAS, P. T. 1940 .

Reproductive versatility in Rubus II. The chromosomes and development.

7. Genet. 40, I 19-1 28.

THOMAS, P. T. 1940 .

The origin of new forms in Rubus III. The chromosome constitution of $R$. loganobaccus, its parents and derivatives.

7. Genet. 40, 1 $4^{1-1} 5^{6}$.

V. WETTSTEIN, F. I 924 .

Morphologie und Physiologie des Formwechsels der Moose auf genetischer Grundlage. I.

Z.I.A.V. 33, 1-236. 